\title{
Difference of coagulation features between severe pneumonia induced by SARS-CoV2 and non-SARS-CoV2
}

\author{
Shiyu Yin ${ }^{1} \cdot$ Ming Huang ${ }^{2} \cdot$ Dengju $\mathrm{Li}^{3} \cdot$ Ning Tang $^{2}$ (i) \\ Published online: 3 April 2020 \\ (c) Springer Science+Business Media, LLC, part of Springer Nature 2020
}

\begin{abstract}
Severe coronavirus disease 2019 (COVID-19) is commonly complicated with coagulopathy, the difference of coagulation features between severe pneumonia induced by SARS-CoV2 and non-SARS-CoV2 has not been analyzed. Coagulation results and clinical features of consecutive patients with severe pneumonia induced by SARS-CoV2 (COVID group) and non-SARS-CoV2 (non-COVID group) in Tongji hospital were retrospectively analyzed and compared. Whether patients with elevated D-dimer could benefit from anticoagulant treatment was evaluated. There were 449 COVID patients and 104 non-COVID patients enrolled into the study. The 28-day mortality in COVID group was approximately twofold of mortality in non-COVID group (29.8\% vs. $15.4 \%, P=0.003)$, COVID group were older $(65.1 \pm 12.0$ vs. $58.4 \pm 18.0$, years, $P<0.001)$ and with higher platelet count $\left(215 \pm 100\right.$ vs. $\left.188 \pm 98, \times 10^{9} / \mathrm{L}, P=0.015\right)$, comparing to non-COVID group. The 28 -day mortality of heparin users were lower than nonusers In COVID group with D-dimer $>3.0 \mu \mathrm{g} / \mathrm{mL}(32.8 \% \mathrm{vs} .52 .4 \%, P=0.017)$. Patients with severe pneumonia induced by SARS-CoV2 had higher platelet count than those induced by non-SARS-CoV2, and only the former with markedly elevated D-dimer may benefit from anticoagulant treatment.
\end{abstract}

Keywords Coronavirus disease $2019 \cdot$ Severe pneumonia $\cdot$ Coagulopathy $\cdot$ D-dimer

\section{Highlights}

Shiyu Yin and Ming Huang contributed equally to the writing of this article.

Ning Tang

tonyjesus@126.com

Shiyu Yin

18963949562@189.cn

Ming Huang

clinicallab2017@126.com

1 Department of Nursing, Tongji Hospital, Tongji Medical College, Huazhong University of Science and Technology, Wuhan, Hubei, China

2 Department of Clinical Laboratory, Tongji Hospital, Tongji Medical College, Huazhong University of Science and Technology, Wuhan, Hubei, China

3 Department of Hematology, Tongji Hospital, Tongji Medical College, Huazhong University of Science and Technology, Wuhan, Hubei, China
- Patients with severe pneumonia induced by SARS-CoV2 had higher platelet count than those induced by nonSARS-CoV2.

- In patients infected by SARS-CoV2 but not non-SARSCov2, those with markedly elevated D-dimer may benefit from anticoagulant treatment.

\section{Introduction}

As recent studies described [1-3], severe coronavirus disease 2019 (COVID-19) is commonly complicated with coagulopathy, markedly elevated D-dimer was associated with poor prognosis of severe COVID-19. The effect of SARS$\mathrm{CoV} 2$ infection on pulmonary coagulation and fibrinolysis is considered to be regulated by various proinflammatory cytokines, and similar to pneumonia induced by other pathogens $[4,5]$. We aimed to compare the coagulation parameters between severe COVID-19 and severe pneumonia induced by other pathogens through retrospective analysis, 
this may be helpful for choosing appropriate treatment on coagulopathy of COVID-19.

\section{Methods}

\section{Patients with severe COVID-19 (COVID group)}

Consecutive patients with severe COVID-19 admitted to Tongji Hospital of Huazhong University of Science and Technology in Wuhan from January 1 to February 13, 2020, were retrospectively enrolled. The medications and outcomes (28-day mortality) were monitored up to March 13, 2020. The diagnosis of COVID-19 was confirmed by RNA detection of the SARS-CoV-2.

\section{Patients with severe pneumonia induced by other pathogens (non-COVID group)}

Consecutive patients with severe pneumonia admitted to intensive care unit of Tongji Hospital from January 1 to October 1, 2019, were retrospectively enrolled. The infection was confirmed by evidence of etiology or imaging.

Exclusion criteria were hospital stay $<7$ days, lack of information about coagulation parameters and medications, and age $<18$ years for both of COVID and Non-COVID groups. A retrospective review of the characteristics of these patients was performed through the electronic medical record system of our hospital. This study was approved by the Ethics Committee of Tongji Hospital (Wuhan, China).

The severe COVID and non-COVID groups were defined as meeting any one of following items, according to the Diagnosis and Treatment Plan of COVID-19 suggested by National Health Commission of China [6]: Respiratory rate $\geq 30$ breaths /min; Arterial oxygen saturation $\leq 93 \%$ at rest; $\mathrm{PaO}_{2} / \mathrm{FiO}_{2} \leq 300 \mathrm{mmHg}$.

As the sepsis-induced coagulopathy (sic.) score system including prothrombin time (PT), platelet count and sequential organ failure assessment (SOFA) has been developed by international society on thrombosis and haemostasis (ISTH) for early recognition and specific treatment of coagulopathy in sepsis patients [7]. Meanwhile, in our previous study [3], higher D-dimer and PT on admission were associated with poor prognosis in patients with COVID-19. Hence these three coagulation parameters D-dimer, PT and platelet count were included in this study and the results were recorded at the time the patients meeting the definition of severe COVID or non-COVID. As an indirect marker of coagulation activation, whether patients with elevated D-dimer could benefit from anticoagulant treatment was evaluated. Anticoagulant treatment was defined as receiving unfractionated heparin (UFH) or low molecular weight heparin (LMWH) for 7 days or longer [8], which was the most commonly used anticoagulant therapy for severe pneumonia in our hospital.

The coagulation tests, including PT and D-dimer were detected using a STA-R MAX coagulation analyzer and original reagents (Diagnostica Stago, Saint-Denis, France). The platelet counts were analyzed by Sysmex XE-2100 haematology analyzer (Sysmex, Kobe, Japan).

Normally and abnormally distributed quantitative variables were compared using the Student's t test and the Mann-Whitney U test, respectively. Categorical variables were compared using the chi-squared test. The results were given as the mean \pm standard deviation, median (interquartile range), or number (percentage), wherever appropriate. A $P$ value of $<0.05$ was considered statistically significant. Data were analyzed using SPSS 21.0 for Windows (SPSS Inc., Chicago, IL, USA).

\section{Results and discussion}

Four hundred and fourty-nine patients classified as COVID group, and 104 patients classified as non-COVID group were enrolled into the study. The clinical characteristics, coagulation results and 28-day mortality between COVID and non-COVID groups were compared (Table 1). The chronic underlying diseases within COVID and non-COVID groups
Table 1 Clinical and coagulation characteristics of COVID and non-COVID groups

\begin{tabular}{llllr}
\hline Parameters & Normal range & COVID $(\mathrm{n}=449)$ & Non-COVID $(\mathrm{n}=104)$ & $P$ values \\
\hline Age (years) & & $65.1 \pm 12.0$ & $58.4 \pm 18.0$ & $<0.001$ \\
Sex ratio (male/female) & & $268 / 181$ & $72 / 32$ & 0.073 \\
With underlying diseases & & $272(60.6 \%)$ & $64(61.5 \%)$ & 0.768 \\
Receiving heparin & $99(22.0 \%)$ & $22(21.2 \%)$ & 0.842 \\
28-day mortality & $134(29.8 \%)$ & $16(15.4 \%)$ & 0.003 \\
Coagulation parameters & & & \\
PT $(\mathrm{sec})$ & $11.5-14.5$ & $15.2 \pm 5.0$ & $16.2 \pm 5.2$ & 0.068 \\
Platelet count & $125-350$ & $215 \pm 100$ & $188 \pm 98$ & 0.015 \\
$(\times 109 / \mathrm{L})$ & & & & \\
D-dimer $(\mu \mathrm{g} / \mathrm{mL})$ & $<0.5$ & $1.94(0.90-9.44)$ & $2.52(1.40-5.81)$ & 0.140 \\
\hline
\end{tabular}


mainly including hypertension, diabetes, heart diseases and lung diseases. The 28-day mortality in COVID group was approximately twofold of mortality in non-COVID group (29.8\% vs. $15.4 \%, P=0.003)$, and COVID group were older $(65.1 \pm 12.0$ vs. $58.4 \pm 18.0$, years, $P<0.001)$ and with higher platelet count $\left(215 \pm 100\right.$ vs $188 \pm 98, \times 10^{9} / \mathrm{L}$, $P=0.015$ ), comparing to non-COVID group.

Ninety-nine (22.0\%) patients of COVID group received heparin treatment for at least 7 days, in which 94 received LMWH (40-60 mg enoxaparin/day) and 5 received UFH (10,000-15,000 U/day); 22 (21.2\%) patients of non-COVID group received heparin treatment, in which 20 received LMWH (40-60 mg enoxaparin/day) and 2 received UFH $(10,000-15,000$ U/day). No anticoagulants other than heparin had been used for 7 days or longer in our patients. All patients received antiviral and appropriate supportive therapies after admission.

No difference on the 28-day mortality was found between heparin users and nonusers in COVID group $(30.3 \%$ vs.
$29.7 \%, P=0.910)$, also in non-COVID group (13.6\% vs. $15.9 \%, P=0.798)$. The association between heparin treatment and outcome in stratified patients according to D-dimer result were evaluated (Fig. 1). When D-dimer exceeding 3.0 $\mu \mathrm{g} / \mathrm{mL}$ (sixfold of upper limit of normal, $6 \mathrm{ULN}$ ), significantly lower mortality in heparin users than nonusers was found in COVID group (32.8\% vs. $52.4 \%, P=0.017$ ). However, no difference on mortality between heparin users than nonusers has been found in non-COVID group when being stratified by D-dimer.

The dysfunction of endothelial cells induced by infection results in excess thrombin generation and fibrinolysis shutdown, which indicated a hypercoagulable state in patient with infection $[9,10]$. In addition, hypoxia existed in severe pneumonia can stimulate thrombosis through not only increasing blood viscosity, but also a hypoxia-inducible transcription factor-dependent signaling pathway [11]. Hence, coagulopathy may be found in quite a lot patients with severe pneumonia.
Fig. 1 Paired bar chart showing the mortality between heparin users and nonusers in patients with severe pneumonia induced by SARS-CoV2 (a COVID group) and non-SARS-CoV2 (b non-COVID group) stratified by D-dimer level. D-D, D-dimer; ULN, upper limit of normal $(0.5 \mu \mathrm{g} / \mathrm{mL})$; ${ }^{*} P<0.05$ between heparin users and nonusers
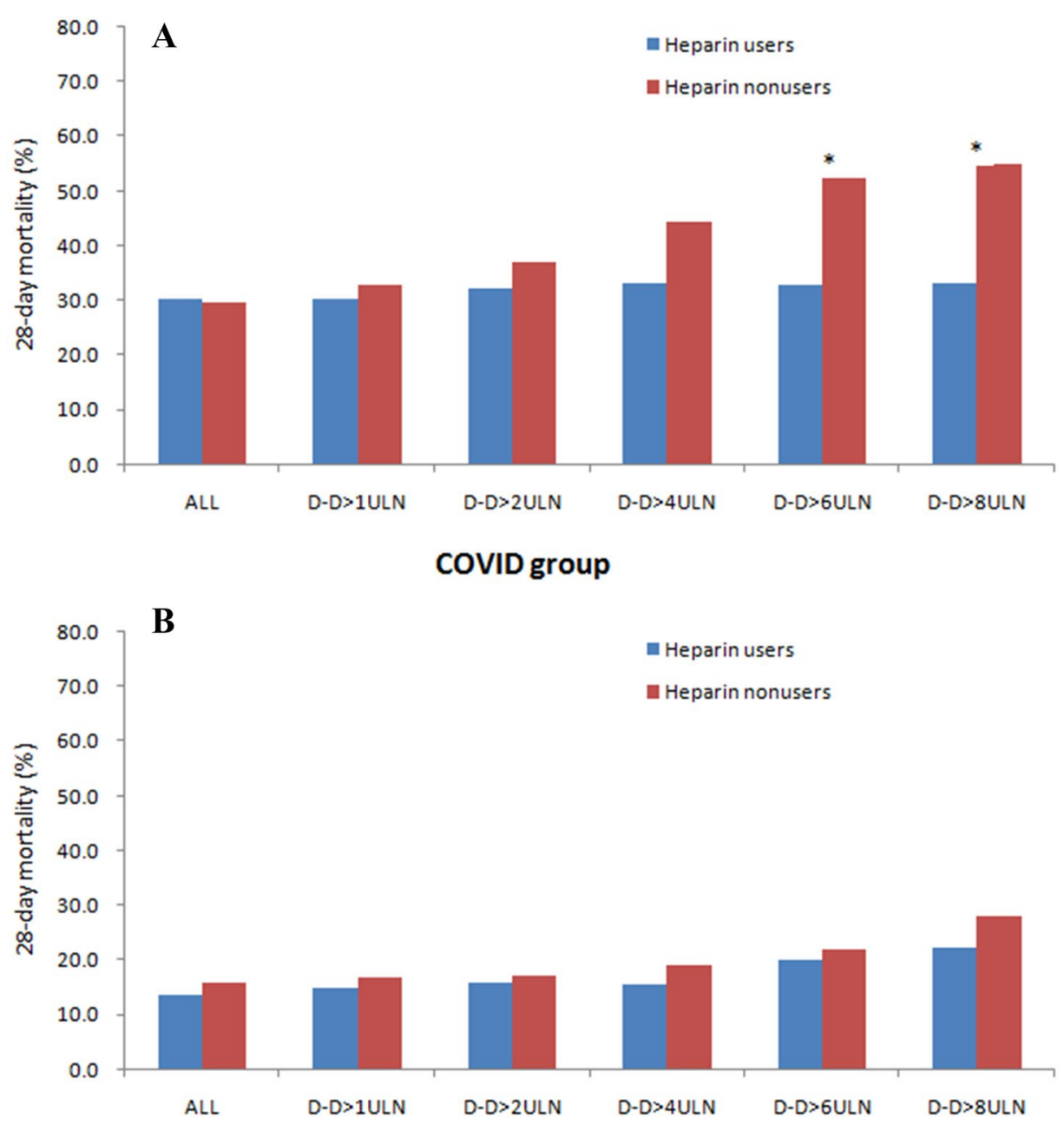

Non-COVID group 
In this study, platelet count of COVID group was significantly higher than that of non-COVID group, perhaps due to the reactively increased thrombopoietin following pulmonary inflammation [12], this might mean more severe inflammation reaction and hypercoagulability in COVID group, and platelet count may not be a sensitive marker for coagulopathy of COVID-19.

LMWH was the most commonly used anticoagulant in our hospital for preventing DIC and VTE in patients, also because of its anti-inflammatory effect [13]. Another reason is that other anticoagulants, such as recombinant soluble thrombomodulin or antithrombin, is unavailable in China yet. However, the effectiveness of anticoagulant therapy for sepsis-associated DIC is still controversial $[14,15]$ our study also suggests that anticoagulant may not benefit to the unselected patients whether in COVID or non-COVID group. Although it has been confirmed that markedly elevated D-dimer was associated with poor prognosis in severe pneumonia [3,5], only COVID group with elevated D-dimer ( $>6$ ULN) could benefit from heparin treatment in our study, this might due to the more obvious hypercoagulability in COVID group, and heterogeneity of coagulation status induced by various pathogens in non-COVID group.

There were several limitations in current study. First, potential selection bias exists in this retrospective study, for instance, LMWH might tend to be used in patients with targeted symptom or medical history, which we have not controlled. Second, the influence of other therapies on these patients has not been evaluated. Nonetheless, this study included a large critical patient populations, we believe that the results of current study still has certain clinical significance.

In conclusion, patients with severe pneumonia induced by SARS-CoV2 had higher platelet count than those induced by non-SARS-CoV2, and only the former with markedly elevated D-dimer may benefit from anticoagulant therapy mainly with LMWH. Further prospective studies are needed to confirm these results.

Author contributions SY drafted the manuscript, SY and MH collected and analyzed the data, DL interpreted the data, NT designed the study.

Funding National Mega Project on Major infectious Disease Prevention of China (No. 2017ZX10103005-007).

\section{Compliance with ethical standards}

Conflict of interest The authors declare that they have no conflicts of interest.

\section{References}

1. Chen N, Zhou M, Dong X et al (2020) Epidemiological and clinical characteristics of 99 cases of 2019 novel coronavirus pneumonia in Wuhan, China: a descriptive study. Lancet 395(10223):507-513

2. Huang C, Wang Y, Li X et al (2020) Clinical features of patients infected with 2019 novel coronavirus in Wuhan, China. Lancet 395(10223):497-506

3. Tang N, Li D, Wang X, Sun Z (20220) Abnormal Coagulation parameters are associated with poor prognosis in patients with novel coronavirus pneumonia. J Thromb Haemost

4. Fung SY, Yuen KS, Ye ZW, Chan CP, Jin DY (2020) A tug-of-war between severe acute respiratory syndrome coronavirus 2 and host antiviral defence: lessons from other pathogenic viruses. Emerg Microbes Infect 9(1):558-570

5. Schultz MJ, Haitsma JJ, Zhang H, Slutsky AS (2006) Pulmonary coagulopathy as a new target in therapeutic studies of acute lung injury or pneumonia - a review. Crit Care Med 34(3):871-877

6. National Health Commission of China (2020) The diagnosis and treatment plan for the novel coronavirus disease, 7 th edn. National Health Commission of China, Bejing

7. Iba T, Nisio MD, Levy JH, Kitamura N, Thachil J (2017) New criteria for sepsis-induced coagulopathy (SIC) following the revised sepsis definition: a retrospective analysis of a nationwide survey. BMJ Open 7(9):e017046

8. Venclauskas L, Llau JV, Jenny JY, Kjaersgaard-Andersen P, Jans $\varnothing$, ESA VTE Guidelines Task Force (2018) European guidelines on perioperative venous thromboembolism prophylaxis: day surgery and fast-track surgery. Eur J Anaesthesiol 35(2):134-138

9. Levi M, van der Poll T (2017) Coagulation and sepsis. Thromb Res 149:38-44

10. Schmitt FCF, Manolov V, Morgenstern J et al (2019) Acute fibrinolysis shutdown occurs early in septic shock and is associated with increased morbidity and mortality: results of an observational pilot study. Ann Intensive Care 9(1):19

11. Gupta N, Zhao YY, Evans CE (2019) The stimulation of thrombosis by hypoxia. Thromb Res 181:77-83

12. Menter DG, Kopetz S, Hawk E et al (2017) Platelet "first responders" in wound response, cancer, and metastasis. Cancer Metastasis Rev 36(2):199-213

13. Poterucha TJ, Libby P, Goldhaber SZ (2017) More than an anticoagulant: do heparins have direct anti-inflammatory effects? Thromb Haemost 117(3):437-444

14. Aikawa N, Shimazaki S, Yamamoto Y et al (2011) Thrombomodulin alfa in the treatment of infectious patients complicated by disseminated intravascular coagulation: subanalysis from the phase 3 trial. Shock 35(4):349-354

15. Liu XL, Wang XZ, Liu XX et al (2014) Low-dose heparin as treatment for early disseminated intravascular coagulation during sepsis: a prospective clinical study. Exp Ther Med 7(3):604-608

Publisher's Note Springer Nature remains neutral with regard to jurisdictional claims in published maps and institutional affiliations. 